Pacific Journal of Mathematics

REGULAR AND IRREGULAR MEASURES ON GROUPS AND

DY ADC SPACES 


\section{REGULAR AND IRREGULAR MEASURES ON GROUPS AND DYADIC SPACES}

\section{H. LeRoy Peterson}

It is generally known that if $X$ is a $\sigma$-compact metric space, then every Borel measure on $X$ is regular. It is not difficult to prove a slightly stronger result, namely that the same conclusion holds if $X$ is a Hausdorff space in which every open subset is $\sigma$-compact (I.6 below). The converse is not generally true, even for compact Hausdorff spaces; a counter-example appears here under IV. 1. However, it will be shown in $\S$ II that every nondegenerate Borel measure on a nondiscrete locally compact group is regular if and only if the group is $\sigma$-compact and metrizable. A similar theorem, proved in § III, holds for dyadic spaces: every Borel measure on such a space is regular if and only if the space is metric.

The result for groups depends on two structure theorems which are proved here: every nonmetrizable compact connected group contains a nonmetrizable connected Abelian subgroup (II.10), and every nonmetrizable locally compact group contains a nonmetrizable compact totally disconnected subgroup (II.11).

In $\S$ III, it seems that the separable case requires special attention: a theorem is proved which has as a corollary that every separable dyadic space is a continuous image of $\{0,1\}^{\circ}$ (III.3 and III.4), and one lemma (III.6) uses a weakened version of the continuum hypothesis.

I. Regular and irregular measures.

1. Let $X$ be a topological space, $\mathbf{M}$ a $\sigma$-algebra of subsets of $X$, and $\mu$ a (countably additive, nonnegative) measure function whose domain is $\mathbf{M}$. The system $(X, \mathbf{M}, \mu)$ is called regular measure space and $\mu$ is called a regular measure in case

(1) $\mu C<\infty$ for all compact $C \in \mathbf{M}$;

(2) $\mu S=\inf \{\mu U: U$ open, $U \in \mathbf{M}, U \supset S\}$ for all $S \in \mathbf{M}$;

(3) $\mu U=\sup \{\mu C: C$ compact, $C \in \mathbf{M}, C \subset U\}$ for all open $U \in \mathbf{M}$.

For lack of a better term, a measure $\mu$ will be called totally regular if it satisfies the more exclusive definition of regularity favored by some authors (e.g., Halmos in [5]), namely:

$$
\begin{aligned}
\mu S & =\sup \{\mu C: C \text { compact, } C \in \mathbf{M}, C \subset S\} \\
& =\inf \{\mu U: U \text { open, } U \in \mathbf{M}, U \supset S\} \text { for all } S \in \mathbf{M} .
\end{aligned}
$$

REMARK 2. According to [7], (10.30) and (10.31), any $\sigma$-finite regular measure on a Hausdorff space is totally regular; the proof as 
given is for Radon measures but almost exactly the same argument will work for any regular measure.

3. A measure $\mu$ will be called irregular if

(1) $\mu$ is not regular;

(2) $\mu C<\infty$ for all compact $C \in \mathbf{M}$;

(3) $\mu$ is nondegenerate: i.e., $\mu$ has values other than 0 and $\infty$.

4. Let $X$ be a topological space. $\mathbf{B}(X)$ is defined to be the smallest $\sigma$-algebra containing the closed subsets of $X$. A Borel measure on $X$ is a measure defined on $\mathbf{B}(X)$ which assigns finite measure to each compact member of $\mathbf{B}(X)$.

5. Note. Research on nonregular measures has appeared in [12], [13], and [14], and examples of irregular Borel measures are to be found in [5] and [7]; see II.2 and IV.2 below.

It is clear that the construction of a nonregular degenerate measure on a space which is not $\sigma$-compact presents no problem: simply assign measure 0 to sets which are contained in $\sigma$-compact sets, and measure $\infty$ to other sets.

Lemma 6. Let $X$ be a topological space such that every open subset of $X$ is the union of countably many closed sets. Let $\mu$ be a $\sigma$-finite Borel measure on $X$. Then

$$
\mu B=\sup \{\mu F: F \text { closed, } F \subset B\}=\inf \{\mu U: U \text { open, } U \supset B\}
$$

for all $B \in \mathbf{B}(X)$.

(This result is due to E. Zakon [16].)

THEOREM 7. Let $X$ be a Hausdorff space. If every open subset of $X$ is $\sigma$-compact, then every Borel measure on $X$ is totally regular.

Proof. This follows easily from the preceding lemma.

COROLlary 8. Every Borel measure on a $\sigma$-compact metric space is regular.

II. Locally compact groups. All topological groups in this section are assumed to be Hausdorff.

THEOREM 1. Let $G$ be a locally compact group which is neither $\sigma$-compact nor discrete. Then $G$ admits an irregular Borel measure.

Proof. Let $\lambda$ be a left Haar measure on $G$. For $B \in \mathbf{B}(G)$, define 
$\nu B=\sup \{\lambda C: C$ compact, $C \subset S\}$. To show that $\nu$ is a nondegenerate Borel measure is a routine exercise. Now let $H$ be an open $\sigma$-compact subgroup of $G$, and let $A$ be a subset of $G$ containing exactly one element of each left coset of $H$. Clearly $A$ is closed and, by the argument in [6], (16.14), $\lambda A=\infty$ but $A$ is locally $\lambda$-null, i.e., $\nu A=0$. Since $\nu U=\infty$ for each neighborhood $U$ of $A, \nu$ is irregular.

[See IV.2 for an example.]

2. Let $Q$ denote the first uncountable ordinal and $\Gamma$ denote an arbitrary ordinal with no countable cofinal subsets, following the standard convention whereby an ordinal is identified with the set of its predecessors.

THEOREM. Let $X_{0}=\Gamma$ with the order topology. For $B \in B\left(X_{0}\right)$, define

$$
\mu B=\left\{\begin{array}{l}
1 \text { if } B \text { contains a closed cofinal subset of } X_{0} \\
0 \text { otherwise }
\end{array}\right.
$$

Then $\mu$ is an irregular Borel measure on $X_{0}$.

Proof. The argument is essentially the same as that required for the special case $\Gamma=\Omega$, which appears as an exercise in [5] (p. 231). Using a variation of the "interlacing lemma" as in [1], it can be shown that the intersection of countably many closed cofinal sets is cofinal; thus a member of $\mathbf{B}\left(X_{0}\right)$ has measure 1 if and only if its complement has measure 0 and the union of countably many sets of measure 0 has measure 0 also, so that $\mu$ is indeed a Borel measure. The measure is irregular as $\mu X_{0}=1$ while $\mu C=0$ for every compact subset $C$ of $X_{0}$.

Corollary 3. Let $X_{1}=\Gamma \cup\{\Gamma\}$ with the order topology and let $X$ be a $T_{1}$ space. Suppose that there is a continuous function $h$ from $X_{1}$ into $X$ such that $h^{-1}\{(h \Gamma)\}$ is not cofinal in $X_{0}$. Then $X$ admits a finite irregular Borel measure.

Proof. It is easy to verify that $h^{-1}(B) \cap X_{0}$ is in $\mathbf{B}\left(X_{0}\right)$ whenever $B$ is in $\mathbf{B}(X)$. Let $\mu$ be the irregular measure defined in II.2 and define $\nu$ on $\mathbf{B}(X)$ by $\nu B=\mu\left(h^{-1}(B) \cap X_{0}\right)$; evidently $\nu$ is a Borel measure, which is irregular since $\nu\{h(\Gamma)\}=0$ but $\nu U=1$ for each neighborhood $U$ of $h(\Gamma)$.

Embedding Theorem 4. Let $X_{1}=\Omega \cup\{\Omega\}$ with the order topology. $X_{1}$ is homeomorphic to a subspace of $\{0,1\}^{\Omega}$ (with the product topology). 
Proof. For $\alpha \in X_{1}$, define $h(\alpha)$ in $\{0,1\}^{2}$ by

$$
[h(\alpha)]_{\beta}=h_{\beta}(\alpha)=\left\{\begin{array}{l}
0 \text { if } \alpha \leqq \beta \\
1 \text { if } \alpha>\beta
\end{array}\right.
$$

Evidently, $h$ is one-to-one. Each coordinate function $h_{\beta}$ is continuous from $X_{1}$ into $\{0,1\}$; thus $h$ is continuous.

CoRollary 5. Any space which contains $\{0,1\}^{\Omega}$ as a closed subspace admits a finite irregular Borel measure.

REMARK 6. According to a theorem of Ivanovskii et. al. ([6], (9.15)), every nonmetrizable compact totally disconnected group is homeomorphic to $\{0,1\}^{m}$ for some uncountable $m$. By II.5, every such group therefore admits an irregular Borel measure; this is a special case of corollary II.12 below. In order to prove II.12 in general, we show that every nonmetrizable locally compact group has a nonmetrizable compact totally disconnected subgroup.

Lemma 7. Let $G$ be a locally compact group with identity $e$ and closed normal subgroup $H$. If $H$ and $G / H$ are both metrizable, then so is $G$.

Proof. This follows from (8.5) of [6], together with the continuity of the natural homomorphism.

LEMMA 8. Let $G$ be a torsion-free Abelian group of rank $r$. Then there exists a subgroup $K$ of $G$ such that $G / K$ is a torsion group and card $(G / K) \geqq r+1$. If $G$ is uncountable, then card $(G / K)=$ $\operatorname{card}(G)$.

Proof. Let $L$ be a maximal independent subset of $G$, let $K_{0}$ be the subgroup generated by $L$, and (using additive notation) let $K=2 K_{0}$. By the maximality of $L, G / K_{0}$ and therefore $G / K$ are torsion. If $\alpha$ and $\beta$ are distinct elements of $L$, then $\alpha \notin K$ and $\alpha-\beta \notin K$ by the independence of $L$. Thus card $(G / K) \geqq \operatorname{card}(L)+1=r+1$. A standard argument (e.g., see [4], p. 32) shows that if $G$ is uncountable then $\operatorname{card}(G)=r$, so that card $(G / K)=\operatorname{card}(G)$.

TheOREm 9. Let $G$ be a nonmetrizable compact connected Abelian group. Then $G$ contains a nonmetrizable compact totally disconnected subgroup.

Proof. Let $\Gamma$ be the dual group of $G ; \Gamma$ is an uncountable discrete torsion-free Abelian group and thus, by the previous lemma, has a 
subgroup $K$ such that $\Gamma / K$ is an uncountable torsion group. Let $H=\{g \in G: \gamma(g)=1$ for all $\gamma \in K\} ; H$ is a subgroup of $G$, topologically isomorphic to the dual group of $\Gamma / K$, and therefore compact, nonmetrizable, and totally disconnected. (See [6], (23.25), (24.26), and (24.15).)

LEMma 10. Let $G$ be a nonmetrizable compact connected group. Then $G$ contains a nonmetrizable compact connected Abelian group.

Proof. Let $H$ be any maximal $A$ belian subgroup of $G$; according to [9], $H$ is connected and every maximal $A$ belian subgroup of $G$ is a conjugate of $H$. Let $V$ be any intersection of countably many neighborhoods of $e$. By [6], (8.7), $V$ contains a compact normal subgroup $N$ of $G$ such that $G / N$ is metrizable. Suppose $N \cap H=\{e\}$; then $N \cap H^{\prime}=\{e\}$, where $H^{\prime}$ is any other maximal Abelian subgroup of $G$. Consequently $N=\{e\}$, which is impossible since $G$ is not metrizable. Thus $V \cap H \supset N \cap H \neq\{e\}$, and thus $H$ is not metrizable.

THeOREM 11. Let $G$ be a nonmetrizable locally compact group. Then $G$ contains a nonmetrizable compact totally disconnected subgroup.

Proof. (1) Assume $G$ is compact. Let $C$ be the component of $e$ in $G$. If $C$ is metrizable, then there exists a compact normal subgroup $H$ of $G$ such that $H \cap C=\{e\}$ and $G / H$ is metrizable; by II.7, $H$ is not metrizable. The natural homomorphism $g \rightarrow g^{C}$ is a topological isomorphism of $H$ onto $C H / C$, a subgroup of the totally disconnected group $G / C$ ([6], (7.3)); $H$ is therefore totally disconnected. If $C$ is not metrizable, then $C$ contains a nonmetrizable compact totally disconnected subgroup, by II.9 and II.10.

(2) Now suppose $G$ is not compact. By part (1), we have only to show that $G$ has a nonmetrizable compact subgroup. Let $H$ be an open compactly generated subgroup; by [6], (8.5) and (8.7), $H$ is not metrizable and has a compact normal subgroup $N$ such that $H / N$ is metrizable, and thus $N$ is not metrizable.

COROLLARY 12. Every nonmetrizable locally compact group admits a finite irregular Borel measure, concentrated on a compact totally disconnected subgroup.

This follows from the remark in II.6. Combining II.12 with II.1 I.8, we have:

THEOREM 13. Let $G$ be a nondiscrete locally compact group. 
Then every nondegenerate Borel measure on $G$ is regular if and only if $G$ is $\sigma$-compact and metrizable.

\section{Dyadic spaces.}

1. A dyadic space is a Hausdorff space which is the image, under a continuous mapping, of $\{0,1\}^{A}$ for some set $A$, where $\{0,1\}$ is discrete and the product has the product topology. According to a standard theorem, every compact metric space is a dyadic space; thus a dyadic space is any Hausdorff space which is a continuous image of a product of compact metric spaces. Recent interesting papers on dyadic spaces include [2] and [3], which contain references to earlier writings.

Theorem 2. Let $X$ be a dyadic space. Then every Borel measure on $X$ is regular if and only if $X$ is metric.

Proof. If $X$ is metric, then every Borel measure on $X$ is regular, by I.8; to prove the converse statement, some preliminary results have to be established, as follows:

Theorem 3. Let $X$ be a dyadic space and $D$ a dense subset of $X$. Then there is a continuous function from $\{0,1\}^{2 D}$ onto $X$.

[See [3], Theorem 1, for related result.]

Proof. Let $f$ be a continuous function from $\{0,1\}^{A}$ onto $X$. Choose $E \subset\{0,1\}^{A}$ such that $f \mid E$ is one-to-one and $f(E)=D$. Define an equivalence relation $\sim$ on $A$ as follows: $\alpha \sim \beta$ in case $x_{\alpha}=x_{\beta}$ for all $x \in E$. Define $u(\alpha)=\left\{x \in E: x_{\alpha}=1\right\}$ and $U=\{u(\alpha): \alpha \in A\}$; clearly $u(\alpha)=u(\beta)$ if and only if $\alpha \sim \beta$. Define a mapping $g$ from $\{0,1\}^{U}$ into $\{0,1\}^{A}$ by $[g(t)]_{\alpha}=g_{\alpha}(t)=t_{u(\alpha)}$ for each $t=\left(t_{u(\alpha)}\right)$ in $\{0,1\}^{U}$ and each $\alpha$ in $A$. Each $g_{\alpha}$ is continuous from $\{0,1\}^{U}$ into $\{0,1)$, thus $g$ is continuous, and $f \circ g$ is a continuous mapping from $\{0,1\}^{c}$ into $X$. The image of $g$ in $\{0,1\}^{A}$ is the set $\left\{x: x_{\alpha}=x_{\beta}\right.$ whenever $\left.\alpha \sim \beta\right\}$, which contains $E$. Thus $f \circ g$ is a continuous function from $\{0,1\}^{c}$ onto a dense compact subset of $X$, which must be $X$ itself. Now card $U$ $\leqq 2^{\operatorname{card} E}=2^{\operatorname{card} D}$; thus there is a continuous function from $\{0,1\}^{2^{D}}$ onto $X$.

Corollary 4. A dyadic space is separable if and only if it is a continuous image of $\{0,1\}^{c}$.

Proof. By [11], Theorem 1, $\{0,1\}^{c}$ (and every continuous image thereof) is separable. The converse follows from III.3. 
THEOREM 5. Let $X$ be a topological space and let $\left\{X_{\alpha}: \alpha<\Gamma\right\}$ be a nondecreasing transfinite sequence of proper closed subsets of $X$ with $\cup X_{\alpha}$ dense in $X$. Let $A$ be a subset of $\Gamma$. Then the following statements are equivalent:

(1) $A$ is cofinal in $\Gamma$.

(2) $\bigcup\left\{X_{\alpha}: \alpha \in A\right\}=\bigcup\left\{X_{\alpha}: \alpha<\Gamma\right\}$.

(3) $\bigcup\left\{X_{n}: \alpha \in A\right\}$ is dense in $X$.

Proof. It is clear that each of (1) and (2) implies the statement following it. To show that (3) implies (1), suppose $A$ has an upper bound $\alpha_{\imath}<\Gamma$. Then $\mathrm{U}\left\{X_{\alpha}: \alpha \in A\right\} \subset X_{\alpha_{0}}$, which is a proper closed subset of $X$, and so $\bigcup\left\{X_{\alpha}: \alpha \in A\right\}$ is not dense in $X$, contradicting (3).

[Note: To prove the next lemma, we assume a weakened version of the continuum hypothesis, namely that $c=\boldsymbol{\aleph}_{j}$ for some $\left.j=1,2, \cdots\right]$.

Lemma 6. Let $X$ be a nonmetric dyadic space. Let $\Gamma$ be the smallest ordinal such that $X$ contains a nonmetric subspace which is a continuous image of $\{0,1\}^{\Gamma}$. Then $\Gamma$ does not have a countable cofinal subset.

Proof. Let $X_{\Gamma}$ be the continuous image of $\{0,1\}^{\Gamma}$ referred to in the hypothesis; $\Gamma$ is uncountable since $X_{\Gamma}$ is not metric. If $X_{\Gamma}$ is separable, then card $(\Gamma) \leqq c$, so by the note above, $\Gamma$ does not have a countable cofinal subset. On the other hand, if $X_{\Gamma}$ is not separable, let $f$ be a continuous function from $\{0,1\}^{\Gamma}$, onto $X_{\Gamma}$ and for $\alpha<\Gamma$ let

$$
F_{\alpha}=\left\{y: y \in\{0,1\}^{\Gamma}, y_{\beta}=0 \text { for all } \alpha \leqq \beta<\Gamma\right\} \text {. }
$$

Let $X_{\alpha}=f\left(F_{\alpha}\right) . \bigcup F_{\alpha}$ is dense in $\{0,1\}^{\Gamma}$ and thus $\bigcup X_{\alpha}$ is dense in $X_{\Gamma}$. Now, for each $\alpha<\Gamma, F_{\alpha}$ is homeomorphic to $\{0,1\}^{\alpha}$, thus $X_{\alpha}$ is a compact metric (hence closed and separable) subspace of $X_{\Gamma}$. Since $X_{\Gamma}$ is not separable, it is impossible for the union of countably many $X_{\alpha}$ to be dense in $X_{\Gamma}$, and therefore by III.5, $\Gamma$ does not have a countable cofinal subset.

Proof of III.2 (Conclusion). Suppose $X$ is a nonmetric dyadic space. Let $\Gamma$ and $X_{r}$ be as in III.6; let $f, F_{\alpha}$, and $X_{\alpha}(\alpha<\Gamma)$ be as defined above. Since $\Gamma$ has no countable cofinal subset, $\bigcup X_{\alpha} \neq X_{\Gamma}$ (by [2], Corollary 1.). Choose $h(\Gamma)$ in $\{0,1\}^{\Gamma}$ such that $f(h(\Gamma)) \notin \bigcup \bigcup X_{\alpha}$. Let $A=\left\{\alpha<\Gamma: h(\Gamma)_{\alpha}=1\right\}$; then we have

$$
\begin{aligned}
h(\Gamma) \in\{0,1\}^{\Gamma}-\bigcup F_{\alpha} & =\bigcap_{\alpha}\left(\{0,1\}^{r}-F_{\alpha}\right) \\
& =\bigcap_{\alpha}\left\{y: y_{\beta}=1 \text { for some } \alpha \leqq \beta<\Gamma\right\},
\end{aligned}
$$


thus $A$ is cofinal in $\Gamma$ and so has no countable cofinal subset. Let $X_{1}=A \cup\{\Gamma\}$ with the well-ordering inherited from $\Gamma \cup\{\Gamma\}$ and with the order topology. Define a function $h$ from $X_{1}$ into $\{0,1\}^{r}$ coordinatewise by

$$
[h(\alpha)]_{\beta}=h_{\beta}(\alpha)=\left\{\begin{array}{l}
1 \text { if } \beta \in A \text { and } \beta<\alpha \\
0 \text { otherwise }
\end{array}\right.
$$

for $\alpha \in A$ and $\beta<\Gamma$; $h(\Gamma)$ has already been defined. By the definition of the topologies of $X_{1}$ and $\{0,1\}$, each coordinate function $h_{\beta}$ is continuous, thus, $h$ is continuous. It is obvious that $h$ is one-to-one. Now $f \circ h$ is a continuous function from $X_{1}$ into $X$, and $(f \circ h)^{-1}(\Gamma)=$ $\{\Gamma\}$, for if $\alpha \in A$ then $f \circ h(\alpha) \in X_{\alpha}$, but $f \circ h(\Gamma)=f(h(\Gamma)) \notin X_{\alpha}$. Since $A$ has no countable cofinal subset, II.3 applies and $X$ admits an irregular Borel measure.

COROLLARY 7. Every nonmetrizable locally compact group admits a finite irregular Borel measure, concentrated on a compact subgroup.

Proof. II.11(2) of this paper shows that a nonmetrizable locally compact group has a nonmetrizable compact subgroup. According to a theorem of Kuzminov, [6] p. 106, every compact group is a dyadic space.

The reader will note that this corollary is a less precise version of II.12.

8. (A concluding remark on finite irregular measures.) A measure $\nu$ is continuous if each point $x \in X$ is an element of a set of measure; $0 ; \nu$ is atomic if it has an atom, i.e., a measurable set $A$, such that $\nu A>0$ and such that, when $S$ is a measurable subset of $A$, either $\nu S=0$ or $\nu S=\nu A$.

THEOREM. Let $X$ be a Hausdorff space and let $(X, \mathbf{M}, \nu)$ be a measure space with $\nu=\nu_{1}+\nu_{2}$, where $\nu_{1}$ is a finite continuous atomic measure. Then $\nu$ is irregular.

Proof. We assume without loss of generality that $\nu_{2}=0$ and $\{x\} \in \mathbf{M}$ for all $x \in X$. Let $A$ be an atom and let $\mathbf{C}=\{C: C \in \mathbf{M}, C$ compact, $C \subset A, \nu C=\nu A\}$. If $\mathrm{C}=\varnothing, \nu$ is irregular, according to I.2. If $\mathbf{C} \neq \varnothing$, then $\mathbf{\cap} \neq \neq \varnothing$ as $\mathbf{C}$ is a collection of closed compact sets with the finite intersection property. Let $x \in \cap \mathrm{C}$. If $C$ is a compact measurable subset of $A-\{x\}$, then $\nu C=0$ as $C \subset A$ and $x \notin C$. But $\nu(A-\{x\})=\nu A>0$, thus $\nu$ is irregular.

It will be noted that the finite irregular Borel measures described 
in $\S$ II and $\S I I I$ are atomic. The author is not aware of any finite irregular measures that do not have the properties described in the theorem above.

\section{Examples.}

1. Let $X$ be the one-point compactification of a discrete space of cardinality $\boldsymbol{\aleph}_{1}$. Evidently, every subset of $X$ is either open or closed (or both), and thus a member of $\mathbf{B}(X)$. Every Borel measure $\mu$ on $X$ is therefore a finite measure defined on all subsets of $X$, and so, by a theorem of Ulam [15],

$$
\begin{aligned}
\mu B & =\sum_{x} \mu(\{x)\} \quad(x \in B) \\
& =\sup \{\mu A: A \text { is finite and } A \subset B\} \\
& =\inf \{\mu S: X-S \text { is finite and } B \subset S\} .
\end{aligned}
$$

Thus $\mu$ is totally regular. However, any uncountable subset of $X-\{\infty\}$ is an open set which is not $\sigma$-compact.

This example provides a comment on II.3; we cannot weaken the hypothesis by eliminating the condition that $h^{-1}\{(h \Gamma)\}$ not be cofinal, even if we substitute the condition that $X$ have non- $\sigma$-compact open subsets. Let $\Gamma=\Omega$ and take $X$ to be the one-point compactification of the isolated ordinals in $\Omega$. Define $h: X_{1} \rightarrow X$ by

$$
h(\alpha)=\left\{\begin{array}{l}
\infty \text { if } \alpha \text { is a limit ordinal } \\
\alpha \text { otherwise }
\end{array}\right.
$$

Then $h$ is continuous but $X$, as just noted, admits no irregular Borel measure.

2. Let $R$ denote the reals with the usual topology and $R_{d}$ the reals with the discrete topology; let $G=R_{d} \times R$ with the product topology. For $r \in R_{d}$ and $S \subset G$, set $S(r)=\{x:(r, x) \in S\}$. Note that if $B \in \mathbf{B}(G)$, then $B(r) \in \mathbf{B}(R)$ for all $r \in R_{d}$. Define

$$
\nu B=\sum_{r} \lambda(B(r)) \quad\left(r \in R_{d}\right)
$$

where $\lambda$ denotes Lebesgue measure on $R$. Clearly $\nu$ is a nondegenerate Borel measure, which is irregular since there is a set $A=R_{d} \times\{0\}$ such that $\nu A=0$ but $\nu U=\infty$ for any neighborhood $U$ of $A$.

This example, which has appeared in [7] (Exercise 12.58), provides a specific illustration for Theorem II.1. For let

$$
\mu S=\inf \{\nu U: U \text { open, } U \supset S\} ;
$$


it can be shown (see [10], 2.22) that $\mu$ is a Haar measure for $G$, and that

$$
\nu B=\sup \{\mu C: C \text { compact, } C \subset B\}
$$

\section{REFERENCES}

1. J. Dieudonné, Un exemple d'espace normal non susceptible d'une structure uniforme d'espace, complet, C. F. Acad. Sci. Paris 209 (1939), 145-147.

2. B. Efimov and R. Engelking, Remarks on dyadic spaces II, Colloq. Math. 13 (1965), $181-197$.

3. R. Engelking and A. Pelczynski, Remarks on dyadic spaces, Colloq. Math. 11 (1963), $55-63$.

4. L. Fuchs, Abelian groups, Pergamon Press, London, 1960.

5. P. R. Halmos, Measure theory, Van Nostrand, Princeton, 1950.

6. E. Hewitt and K. A. Ross, Abstract harmonic analysis I, Springer-Verlag, Berlin, 1963.

7. E. Hewitt and K. R. Stromberg, Real and abstract analysis, Springer-Verlag, New York, 1965.

8. J.L. Kelley, General topology, Van Nostrand, Princeton, 1955.

9. J. Mycielski, Some properties of connected compact groups, Colloq. Math. 5 (1958), $162-166$.

10. H. L. Peterson, Regular and irregular Borel measures, thesis, University of Oregon, 1966.

11. K. A. Ross and A. H. Stone, Products of separable spaces, Amer. Math. Monthly 71 (1964), 389-403.

12. K. A. Ross and K. R. Stromberg, Baire sets and Baire measures, Ark. Mat. 6 (1965), 151-160.

13. G. Swift, Irregular Borel measures on topological spaces, Duke Math. J. 22 (1955), 427-433.

14. —, n-valued irregular Borel measures, Duke Math. J. 23 (1956), 393-407.

15. S. M. Ulam, Zur masstheorie in der allgemeinen Mengenlehre, Fund. Math. 16 (1930), 141-150.

16. E. Zakon, A note on regular measures, Canad. Math. Bull. 7 (1964), 41-44.

Received March 26, 1968. The material in this paper has appeared in the author's $\mathrm{Ph}$. D. thesis (University of Oregon, 1966), written under the supervision of Professor Karl R. Stromberg.

The University of CONNECTICUT 


\section{PACIFIC JOURNAL OF MATHEMATICS}

\section{EDITORS}

H. ROYDEN

Stanford University

Stanford, California

R. R Phelps

University of Washington

Seattle, Washington 98105
J. DUGUNDJI

Department of Mathematics

University of Southern California

Los Angeles, California 90007

RICHARD ARENS

University of California

Los Angeles, California 90024

\section{ASSOCIATE EDITORS}
E. F. BECKENBACH
B. H. NeumanN
F. WOLF
K. YosidA

\section{SUPPORTING INSTITUTIONS}

UNIVERSITY OF BRITISH COLUMBIA

CALIFORNIA INSTITUTE OF TECHNOLOGY

UNIVERSITY OF CALIFORNIA

MONTANA STATE UNIVERSITY

UNIVERSITY OF NEVADA

NEW MEXICO STATE UNIVERSITY

OREGON STATE UNIVERSITY

UNIVERSITY OF OREGON

OSAKA UNIVERSITY

UNIVERSITY OF SOUTHERN CALIFORNIA
STANFORD UNIVERSITY

UNIVERSITY OF TOKYO

UNIVERSITY OF UTAH

WASHINGTON STATE UNIVERSITY

UNIVERSITY OF WASHINGTON

AMERICAN MATHEMATICAL SOCIETY CHEVRON RESEARCH CORPORATION TRW SYSTEMS

NAVAL WEAPONS CENTER 


\section{Pacific Journal of Mathematics

Vol. 28, No. $1 \quad$ March, 1969

Patrick Robert Ahern, On the geometry of the unit ball in the space of real annihilating measures .............................. 1

Kirby Alan Baker, Equational classes of modular lattices ............. 9

E. F. Beckenbach and Gerald Andrew Hutchison, Meromorphic minimal surfaces ......................................... 17

Tae Ho Choe, Intrinsic topologies in a topological lattice ..............

John Bligh Conway, A theorem on sequential convergence of measures and

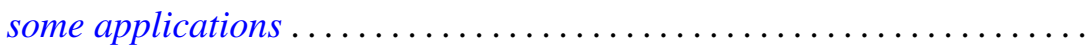

Roger Cuppens, On the decomposition of infinitely divisible probability laws without normal factor.

Lynn Harry Erbe, Nonoscillatory solutions of second order nonlinear

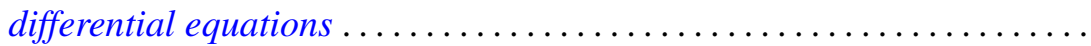

Burton I. Fein, The Schur index for projective representations of finite

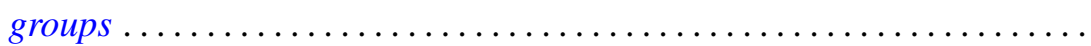

Stanley P. Gudder, A note on proposition observables............... 101

Kenneth Kapp, On Croisot's theory of decompositions ............... 105

Robert P. Kaufman, Gap series and an example to Malliavin's theorem . . . 117

E. J. McShane, Robert Breckenridge Warfield, Jr. and V. M. Warfield,

Invariant extensions of linear functionals, with applications to measures and stochastic processes ................

Marvin Victor Mielke, Rearrangement of spherical modifications ...

Akio Osada, On unicity of capacity functions ..............

Donald Steven Passman, Some 5/2 transitive permutation groups ...

Harold L. Peterson, Jr., Regular and irregular measures on groups and dyadic spaces...

Habib Salehi, On interpolation of $q$-variate stationary stochastic processes...

Michael Samuel Skaff, Vector valued Orlicz spaces generalized

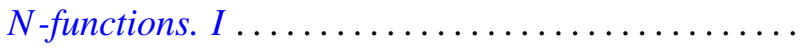

A. J. Ward, On $H$-equivalence of uniformities. II...........

Thomas Paul Whaley, Algebras satisfying the descending chain condition for subalgebras...

G. K. White, On subgroups of fixed index

Martin Michael Zuckerman, A unifying condition for implications among the axioms of choice for finite sets ................. 